\title{
Impact of the tumor microenvironment in predicting postoperative hepatic recurrence of pancreatic neuroendocrine tumors
}

\author{
SHOKI SATO, TAKAHIRO TSUCHIKAWA, TORU NAKAMURA, NAGATO SATO, EIJI TAMOTO, \\ KEISUKE OKAMURA, TOSHIAKI SHICHINOHE and SATOSHI HIRANO \\ Department of Gastroenterological Surgery II, Hokkaido University Graduate School of Medicine, \\ Sapporo, Hokkaido 060-0815, Japan
}

Received August 6, 2014; Accepted September 17, 2014

DOI: $10.3892 /$ or.2014.3530

\begin{abstract}
The disease frequency of pancreatic neuroendocrine tumors (PNETs) has been growing, and postoperative hepatic recurrence (PHR) is one of the factors affecting patient prognosis. The present study aimed to investigate biomarkers of PNETs in the primary disease site to predict PHR using immunohistochemical analysis for tumor-infiltrating lymphocytes (TILs: CD3, CD8 and CD45RO), human leukocyte antigen (HLA) class I, $\alpha$-thalassemia/mental retardation X-linked (ATRX), death domain-associated protein (DAXX), mammalian target of rapamycin (mTOR) and phospho-mTOR (p-mTOR). Correlations were analyzed between TILs and the biomarkers, clinicopathological features and prognosis. Sixteen patients with PNETs who underwent radical surgery at our hospital were reviewed. We analyzed the correlation between PHR and immunohistochemical characteristics, and also between disease-free survival (DFS) or overall survival (OS) and the immunohistochemical characteristics. We found that PHR was associated with the expression patterns of DAXX and p-mTOR. No association was found between PHR and patient background, TILs or other biomarkers. DFS was found to be associated with ATRX, DAXX and p-mTOR. OS was associated only with p-mTOR. In conclusion, ATRX, DAXX and p-mTOR are useful molecular biomarkers for predicting PHR in patients who undergo radical surgery for PNETs. Use of these biomarkers will enable earlier decisions on which patients may benefit from adjuvant therapy.
\end{abstract}

\section{Introduction}

The disease frequency of neuroendocrine tumors has been growing throughout the world. Pancreatic neuroendocrine

Correspondence to: Dr Shoki Sato, Department of Gastroenterological Surgery II, Hokkaido University Graduate School of Medicine, N-15 W-17, Sapporo, Hokkaido 060-0815, Japan

E-mail:a02m033@yahoo.co.jp

Key words: pancreatic neuroendocrine tumor, postoperative hepatic recurrence, tumor-infiltrating lymphocytes, ATRX/DAXX, p-mTOR tumors (PNETs) are one type of neuroendocrine tumors, and their frequency has also been increasing (1). PNETs account for $2 \%$ of all pancreatic neoplasms (1). Although some variants of PNETs exist and often induce hormone hypersecretion and symptoms, PNETs are characteristically slow-growing tumors compared with pancreatic carcinoma. Owing to the multimodal treatment strategy for PNETs, including surgical resection, transcatheter arterial chemoembolization (TACE) and molecular targeting inhibitors, the estimated 5- and 10-year overall survival (OS) after surgical resection have been comparatively favorable, reported as $\sim 65$ and $45 \%$, respectively (2).

However, hepatic recurrence is a factor that affects patient prognosis $(3,4)$. Bloomston et al reported that the liver is the most common site of recurrence, with liver recurrence occurring in $16.7 \%$ of patients following resection of the primary site of PNETs (5). It is important to predict asynchronous hepatic metastasis or recurrence based on clinicopathological characteristics to allow earlier treatment with adjuvant therapy after primary site resection.

Some investigators have analyzed tumor-infiltrating lymphocytes (TILs) in many types of tumor (such as colorectal cancer and hepatocellular carcinoma), and have suggested a correlation between TILs and disease-free survival (DFS) and/or OS (6,7). Therefore, analyzing the local immune response at the primary site of PNETs may predict the risk for hepatic recurrence following curative $\mathrm{R} 0$ resection. Jiao et al investigated exome sequencing in sporadic PNETs for other biomarkers, and identified the presence of somatic mutations in the following genes: multiple endocrine neoplasia-1 (MEN1), death domain-associated protein $(D A X X), \alpha$ thalassemia/ mental retardation $\mathrm{X}$-linked $(A T R X)$ and phosphatase and tensin homolog $(P T E N)$. Moreover, patients who had somatic mutations in MEN1 and/or DAXX/ATRX had better prognosis than patients who had wild-type alleles (8). Mammalian target of rapamycin (mTOR) is another key molecule for predicting patient prognosis. Some investigators have analyzed the expression of mTOR in PNETs. mTOR is involved with the highly conserved 3-kinase-related protein kinase and plays a key role in cellular growth, survival, metabolism and development $(9,10)$; mTOR is activated by phosphorylation. Everolimus, which is one of the molecular targeted inhibitors of mTOR for anti-PNET therapy, improved progression-free 
survival in patients with advanced PNETs as compared with a placebo (11).

The aim of the present study was to investigate tumor biomarkers at the primary disease site that could be used to predict postoperative hepatic recurrence (PHR) of PNETs. Our investigation used immunohistochemical analysis for TILs (CD3, CD8 and CD45RO), human leukocyte antigen (HLA) class I, ATRX, DAXX, mTOR and phospho-mTOR (p-mTOR). The other aim was to analyze the correlations between the clinicopathological features and PNET patient prognosis.

\section{Materials and methods}

Patients. We retrospectively reviewed 24 patients with PNETs who underwent radical surgery at the Department of Gastroenterological Surgery II, Hokkaido University Hospital between 2003 and 2010. We excluded patients who had the following conditions: presence of distant metastasis upon preoperative examination, such as computer tomography (CT), ultrasonography (US), and magnetic resonance imaging (MRI); previous treatment (surgery, chemotherapy or radiation therapy) for PNETs; and/or 9 month or less follow-up period. The reason for this period was that the median period of appearance of PHR was 10 months after surgery at our department. After surgical treatment, the patients underwent follow-up examinations to detect hepatic recurrence including CT and/or US every 6 months.

Clinical tumor typing was determined according to the 2010 World Health Organization (WHO) classification (12). All specimens were fixed in $10 \%$ formalin and embedded in paraffin wax. The thickest part of each tumor was selected for evaluation. Serial $4-\mu \mathrm{m}$-thick sections were obtained and examined by immunohistochemistry. Informed patient consent for immunohistochemical staining was obtained in accordance with the guidelines of the Hokkaido University Institutional Review Board authorization for the present study.

Antibodies. The mouse monoclonal primary antibody used was EMR8-5, which recognizes the heavy chains of HLA-A, HLA-B and HLA-C (clone EMR8-5; Hokudo, Sapporo, Japan) at a 1:1,000 dilution in phosphate-buffered saline (PBS); human CD3-specific monoclonal antibody (mAb) and human CD8-specific mAb (Histofine ${ }^{\circledR}$ CD3 mouse IgG1 $\mathrm{mAb}$ and CD8, mouse IgGlj mAb; Nichirei, Tokyo, Japan) at non-dilution; and anti-CD45RO antibody (UCH-L1; Abcam, Cambridge, UK) at a 1:2,000 dilution were used.

The rabbit monoclonal primary antibody used was mTOR rabbit $\mathrm{mAb}(\# 2983)$ at a 1:50 dilution, and phospho-mTOR rabbit mAb (\#2475) (both from Cell Signaling Technology, Boston, MA, USA) at a 1:100 dilution.

The rabbit polyclonal antibody used was anti-ATRX (HPA001906) at a 1:200 dilution in PBS; and anti-DAXX (HPA008736) (both from Atlas Antibodies AB, Stockholm, Sweden) at a 1:200 dilution in PBS.

Immunohistochemical staining. Immunohistochemical reactions were carried out using the streptavidin-biotin-peroxidase method. As positive controls, normal adenoid tissue was used for CD3, CD8 and CD45RO. Normal mucosal tissues were used for HLA class I heavy chains as internal positive controls. Lung cancers were used for mTOR and p-mTOR, normal pancreas specimens for ATRX, and urinal bladder specimens for DAXX as positive controls. Sections were deparaffinized in xylene, washed in PBS ( $\mathrm{pH} 7.4$ ), and rehydrated in a graded series of ethanol solutions. Antigen retrieval was performed by heating citrate buffer $(\mathrm{pH} 6.0)$ at $120^{\circ} \mathrm{C}$ for $15 \mathrm{~min}$. Endogenous peroxidase activity was blocked by $10-\mathrm{min}$ incubation with $3 \%$ hydrogen peroxide in methanol. After washing in PBS, the specimens were saturated with $10 \%$ normal goat serum (Histofine ${ }^{\circledR}$ SAB-PO kit; Nichirei, Tokyo, Japan) for 5 min and stained with the primary antibody at $4^{\circ} \mathrm{C}$ overnight. After washing in PBS, a biotinylated goat anti-mouse immunoglobulin antibody (Histofine ${ }^{\circledR}$ SAB-PO kit) was applied for $30 \mathrm{~min}$ at room temperature. Immunohistochemical reactions were visualized with freshly prepared 3,3'-diaminobenzidine tetrahydrochloride (Histofine ${ }^{\circledR}$ SAB-PO kit). Slides were counterstained with hematoxylin and mounted on coverslips. All the specimens were evaluated by two investigators blinded to the patient clinical information under the instruction of a pathologist.

Scoring of HLA class I expression status. HLA class I heavy chain expression in the tumor cells was scored using a scale of negative, heterogeneous or positive, when the percentage of stained tumor cells was $<25,25-75$ or $>75 \%$, respectively.

Scoring of $\mathrm{CD}^{+} \mathrm{T}$ cells and $\mathrm{CD}^{+} \mathrm{T}$ cells, and $\mathrm{CD} 45 \mathrm{RO}^{+}$ infiltration. Immunohistochemistry testing and the evaluation of immune cells were performed according to a previous report (13). Briefly, the degree of immune cell infiltration was analyzed in $>10$ independent high-power (x200) microscopic fields for each tissue sample. The numbers of $\mathrm{CD}^{+} \mathrm{T}$ cells, $\mathrm{CD}^{+} \mathrm{T}$ cells and $\mathrm{CD} 45 \mathrm{RO}^{+}$cells were counted in the tumor cell nests.

Scoring of ATR $X, D A X X, m T O R$ and $p$-mTOR. For both ATRX and DAXX scoring, nuclear protein staining was only considered positive in at least $5 \%$ of tumor cells with nuclear labeling, as in a past report (14). Tumor cells were scored as negative if the pattern was that of cytoplasmic accumulation with nuclear clearing, as long as adequate internal controls (for example, nuclear labeling of adjacent endothelial cells, lymphocytes and/or islets of Langerhans) were present (14). For mTOR and p-mTOR scoring, the tissues with $>10 \%$ cytoplasmic- and/or membranous-stained tumor cells were considered positive and the others were negative.

Statistical analysis. All statistical analyses were performed using StatFlex 6.0 software (Artech Company, Osaka, Japan). The Mann-Whitney U test, the Chi-square and Fisher's exact probably tests were applied for comparisons. The durations of DFS and OS were calculated from the date of diagnosis until recurrence or death or until the date of the last follow-up visit for patients still alive. Survival was estimated according to the Kaplan-Meier product limit method. Survival curves were compared using the log-rank test. Results were considered statistically significant when $\mathrm{P}<0.05$ was obtained. 
Table I. Background characteristics of the 16 study patients.

\begin{tabular}{|c|c|c|c|}
\hline \multirow[b]{2}{*}{ Clinical feature } & \multicolumn{2}{|c|}{$\begin{array}{l}\text { Postoperative hepatic } \\
\text { recurrence }\end{array}$} & \multirow[b]{2}{*}{ P-value } \\
\hline & $\begin{array}{l}\text { Positive } \\
\mathrm{n}=5\end{array}$ & $\begin{array}{l}\text { Negative } \\
n=11\end{array}$ & \\
\hline \multicolumn{4}{|l|}{ Gender } \\
\hline Male/female & $2 / 3$ & $4 / 7$ & $1.00^{\mathrm{a}}$ \\
\hline Age, years (range) & $61(35-75)$ & $61(31-72)$ & $0.82^{\mathrm{b}}$ \\
\hline $\begin{array}{l}\text { Tumor size } \\
\text { (mm, range) }\end{array}$ & $35(3.5-70)$ & $13(7-40)$ & $0.23^{\mathrm{b}}$ \\
\hline Hormonal function & & & $0.77^{\mathrm{a}}$ \\
\hline Insulinoma & 0 & 2 & \\
\hline Glucagonoma & 0 & 1 & \\
\hline PPoma & 0 & 1 & \\
\hline $\begin{array}{l}\text { Nonfunctional } \\
\text { tumor }\end{array}$ & 5 & 7 & \\
\hline WHO grade $1 / 2,3$ & $1 / 4$ & $8 / 3$ & $0.105^{\mathrm{a}}$ \\
\hline Surgery & & & $1.00^{\mathrm{a}}$ \\
\hline PD & 2 & 3 & \\
\hline DP & 3 & 5 & \\
\hline DPPHR & 0 & 2 & \\
\hline Other & 0 & 1 & \\
\hline $\begin{array}{l}\text { Follow-up period } \\
\text { (months, range) }\end{array}$ & $39(11-86)$ & $54(17-96)$ & $0.50^{\mathrm{b}}$ \\
\hline Death & & & $0.017^{\mathrm{a}}$ \\
\hline Yes & 3 & 0 & \\
\hline No & 2 & 11 & \\
\hline
\end{tabular}

aFisher's exact probably test; ${ }^{\mathrm{b}} \mathrm{Mann}-\mathrm{Whitney} \mathrm{U}$ test. PPoma, pancreatic polypeptide-producing tumor; PD, pancreaticoduodenectomy; DP, distal pancreatectomy; DPPHR, duodenum preserving pancreatic head resection.

\section{Results}

Background characteristics. The 16 of the 24 patients who underwent radical surgery were included in the present study. (Among the patients excluded, 5 had distant metastasis, 1 had received previous treatment and 2 had been followed up for only two months, postoperatively). The characteristics of the 16 study patients are summarized in Table I. Of the 16 patients who were reviewed, 5 patients had PHR during the follow-up period (PHR-positive), and 11 did not (PHR-negative). There were no statistically significant differences in terms of the preoperative background characteristics between the PHR-positive and the PHR-negative group. The median follow-up period was 39 months ranging 11-86 months in the PHR-positive group, and 54 months ranging 17-96 months in the PHR-negative group $(\mathrm{P}=0.50)$. There was a statistically significant difference between groups in terms of death $(\mathrm{P}=0.017)$. Only the PHR-positive group included deaths; one patient died of interstitial pneumonia and two died of PNETs. There was no significant difference between the two groups
Table II. Comparison of ATRX, DAXX, m-TOR, p-mTOR and HLA class I expression patterns between the PHR-positive and PHR-negative group.

\begin{tabular}{|c|c|c|c|}
\hline \multirow[b]{2}{*}{ Expression patterns } & \multicolumn{2}{|c|}{$\begin{array}{l}\text { Postoperative hepatic } \\
\text { recurrence }\end{array}$} & \multirow[b]{2}{*}{ P-value } \\
\hline & $\begin{array}{l}\text { Positive } \\
n=5\end{array}$ & $\begin{array}{l}\text { Negative } \\
n=11\end{array}$ & \\
\hline ATRX nuclear labeling & & & $0.063^{\mathrm{a}}$ \\
\hline Positive & 2 & 10 & \\
\hline Negative & 3 & 1 & \\
\hline DAXX nuclear labeling & & & $0.036^{\mathrm{a}}$ \\
\hline Positive & 1 & 9 & \\
\hline Negative & 4 & 2 & \\
\hline mTOR expression & & & $0.59^{\mathrm{a}}$ \\
\hline Positive & 4 & 6 & \\
\hline Negative & 1 & 5 & \\
\hline p-mTOR expression & & & $0.036^{\mathrm{a}}$ \\
\hline Positive & 4 & 2 & \\
\hline Negative & 1 & 9 & \\
\hline $\begin{array}{l}\text { HLA class I expression } \\
\text { pattern }(\%)\end{array}$ & & & $0.097^{\mathrm{b}}$ \\
\hline$>75$ & 1 & 3 & \\
\hline $25-75$ & 2 & 4 & \\
\hline$<25$ & 2 & 4 & \\
\hline
\end{tabular}

in terms of the grade of WHO classification (PHR-positive vs. - negative; $\mathrm{P}=0.105)$.

Immunohistochemistry for immune-related cells. $\mathrm{CD}^{+}$ $\mathrm{T}$ cells, $\mathrm{CD}^{+} \mathrm{T}$ cells and $\mathrm{CD} 45 \mathrm{RO}^{+} \mathrm{T}$ cells were detected within cancer cell nests. Representative images of immunohistochemical staining of CD4, CD8 and CD45RO are shown in Fig. 1A-C. The HLA class I expression pattern was classified into three groups; $<25,25-75$ and $>75 \%$ (Fig. 1D-F). Expression patterns of ATRX, DAXX, mTOR and p-mTOR are shown in Fig. 2.

Degree of immune cell infiltration in the tumors and correlation with PHR. We analyzed the infiltration status of CD4, CD8 and CD45RO (Fig. 3) between the PHR-positive and -negative group, and there were no statistically significant differences ( $\mathrm{P}=0.23,0.23$ and 0.28 , respectively). In the HLA class I expression pattern, there was no correlation with TILs (data not shown).

Expression of biomarkers and correlation with PHR. Among all PNETs, the positive rates for ATRX, DAXX, mTOR and p-mTOR were $75.0 \%(12 / 16), 52.5 \%(10 / 16), 52.5 \%(10 / 16)$ and $37.5 \%(6 / 16)$, respectively. The correlations were analyzed between the expression levels of the biomarkers (ATRX, DAXX, mTOR and p-mTOR) and PHR (Table II). There were 

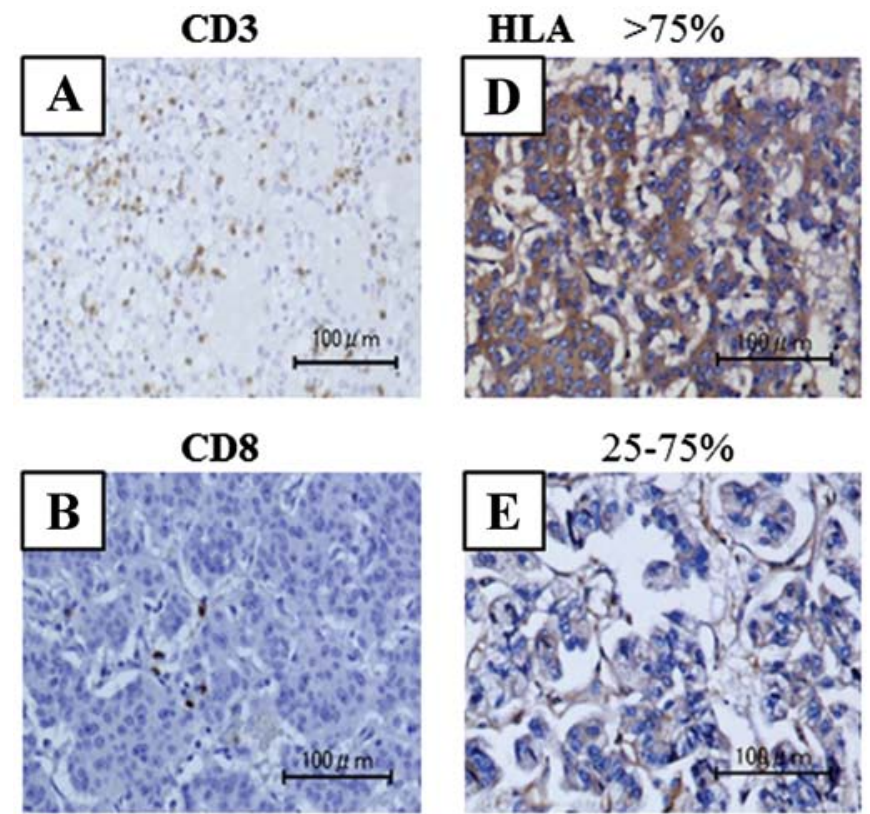

\section{CD45RO}

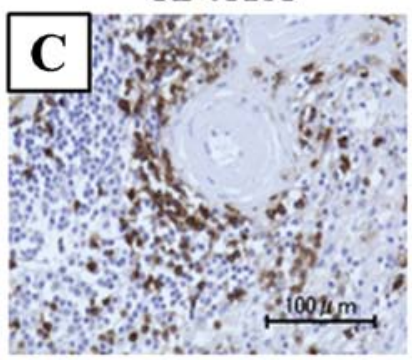

$$
<25 \%
$$

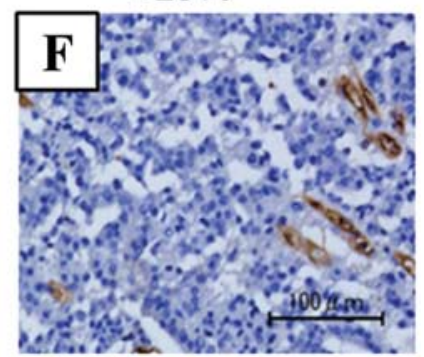

Figure 1. Representative immunohistochemical staining patterns of formalinfixed, paraffin-embedded sections for (A) CD3, (B) CD8 and (C) CD45RO specific monoclonal antibodies. The HLA class I expression pattern was classified into the following three groups: (D) positive, (E) heterogeneous or (F) negative, when the percentage of stained tumor cells was $>75,25-75$ or $<25 \%$, respectively. Scale bar, $100 \mu \mathrm{m}$.

correlations between DAXX-negative and p-mTOR-positive statuses and PHR-positive patients $(\mathrm{P}=0.036$ and $\mathrm{P}=0.036$, respectively).

Correlations between TILs and expression levels of the biomarkers, and prognosis. In DFS, there was no correlation with TILs (data not shown). However, there were correlations with three biomarkers (ATRX, DAXX and p-mTOR; $\mathrm{P}=0.020$, 0.040 and 0.032 , respectively); patients with ATRX-negative, DAXX-negative, or p-mTOR-positive PNETs experienced worse prognosis (Fig. 4). In OS, there was a correlation with p-mTOR only ( $\mathrm{P}=0.029$, Fig. 5).

\section{Discussion}

Several studies have been conducted to investigate the prognosis of patients suffering from PNETs. These studies have demonstrated useful clinical indicators such as tumor size, WHO grade, TNM stage and lymph node metastasis $(15,16)$. Recently, clinicopathological and molecular biomarkers such as TILs, PTEN and p-mTOR have been found useful for estimating the prognosis of patients with PNETs $(17,18)$. However,
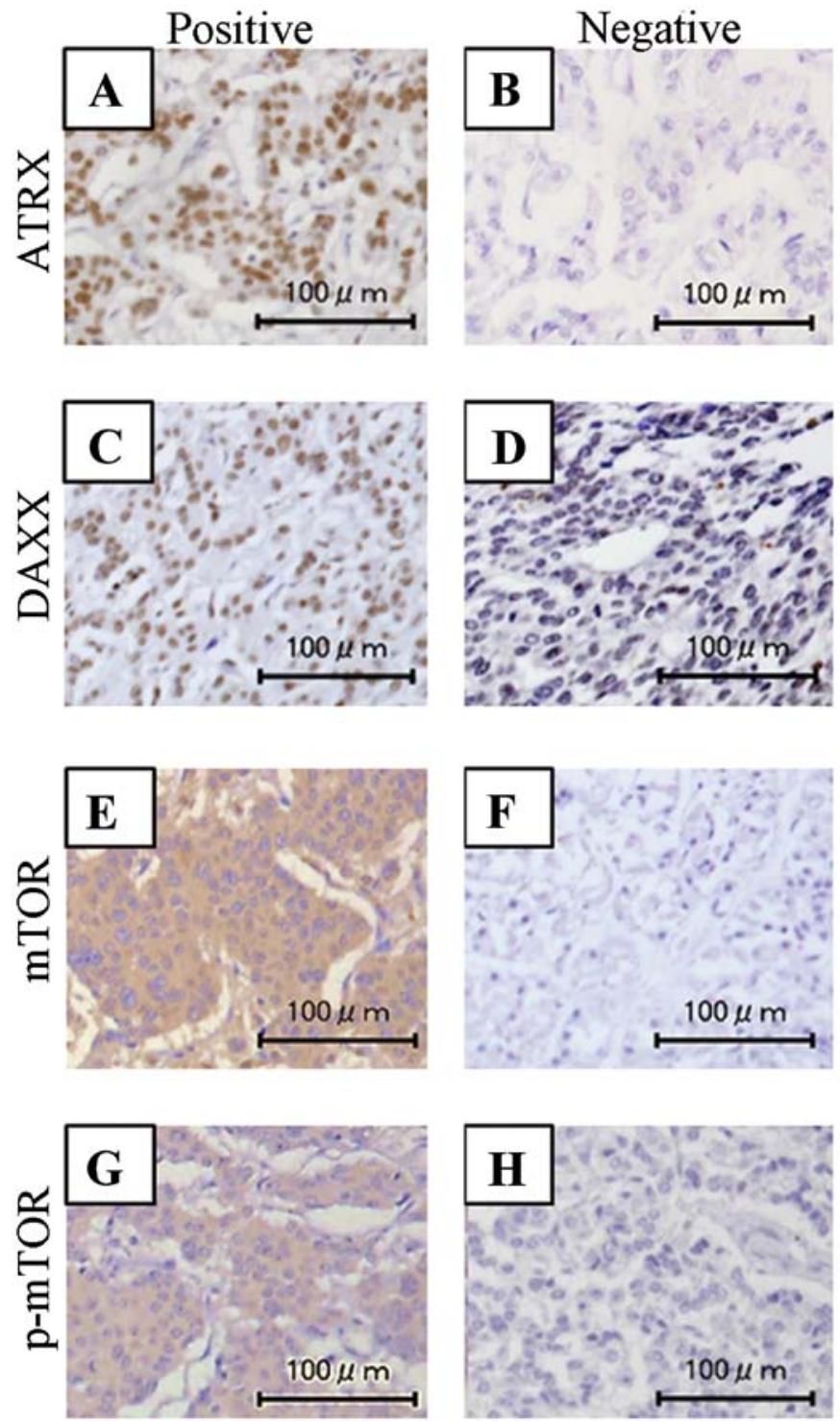

Figure 2. Representative immunohistochemical staining patterns of formalin-fixed, paraffin-embedded sections for ATRX, DAXX, mTOR and p-mTOR. (A) An ATRX-positive sample. Nuclear protein staining was observed. (B) An ATRX-negative sample. (C) A DAXX-positive sample. (D) A DAXX-negative sample. (E) An mTOR-positive sample. Cytoplasmic and/or membranous staining was observed. (F) An mTOR-negative sample. (G) A p-mTOR-positive sample. (H) A p-mTOR-negative sample. Scale bar, $100 \mu \mathrm{m}$.

the primary disease sites of these studies were not unique with respect to the various origins of the neuroendocrine tumors with distant metastasis. In the present study, only patients undergoing surgical resection without residual tumor were included in the investigation of correlations between TILs or other biomarkers and occurrence of PHR.

The present study found no correlation between the number of TILs at the primary disease site and PHR, DFS or OS. Katz et al reported that the degree of infiltration by FoxP3-positive TILs could predict OS following treatment of the neuroendocrine tumor liver metastasis nest, while $\mathrm{CD}^{+}$, $\mathrm{CD}^{+}$and $\mathrm{CD} 8^{+}$TILs could not (17). Yet, the primary sites of the metastasis were not only the pancreas but also the small bowel and other in the present study. In pancreatic ductal 


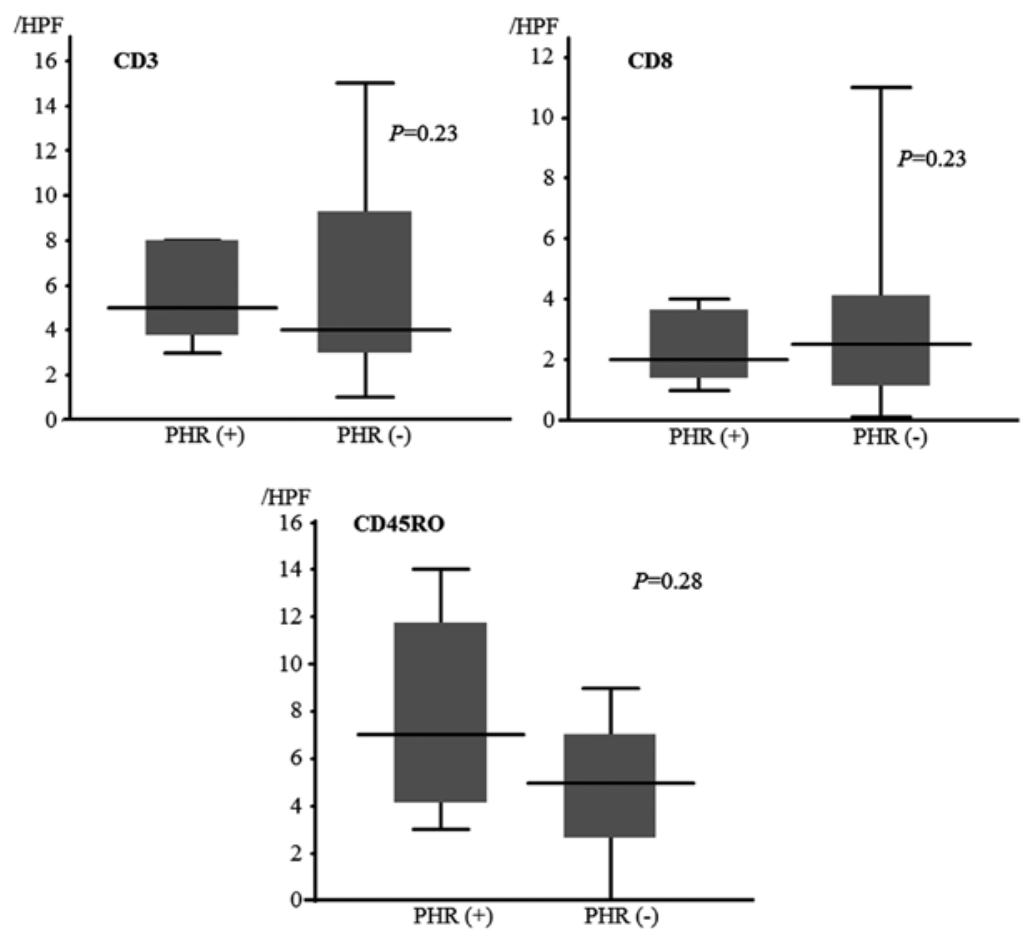

Figure 3. The degree of immune cell infiltration in the tumor cell nest of PNETs. We found no significant difference in the number of $\mathrm{CD}^{+}, \mathrm{CD}^{+}$or $\mathrm{CD} 45 \mathrm{RO}$ $\mathrm{T}$ cells between the PHR-positive and PHR-negative group.
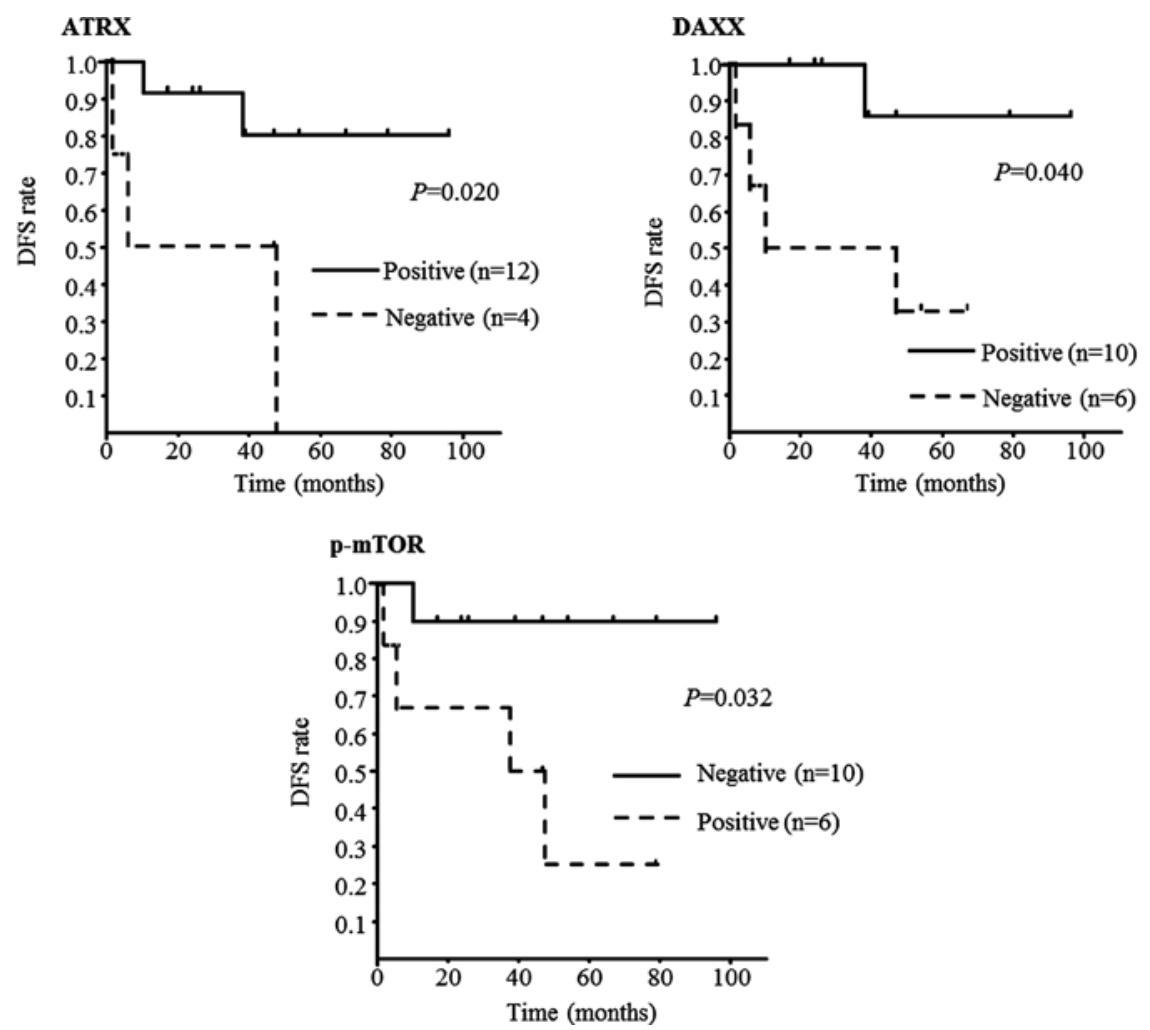

Figure 4. Disease-free survival (DFS) in accordance with the presence of ATRX, DAXX and p-mTOR. ATRX-negative, DAXX-negative and p-mTOR-positive PNET patients had the least favorable prognosis. PNETs, pancreatic neuroendocrine tumors.

cancer, tumor-infiltrating $\mathrm{CD}^{+}, \mathrm{CD}^{+}$, regulatory $\mathrm{T}$ cells (Treg) and tumor-infiltrating macrophages (type 1 and 2) have been reported as independent prognosticators (19). The HLA expression pattern of PNETs in the present study was lower 


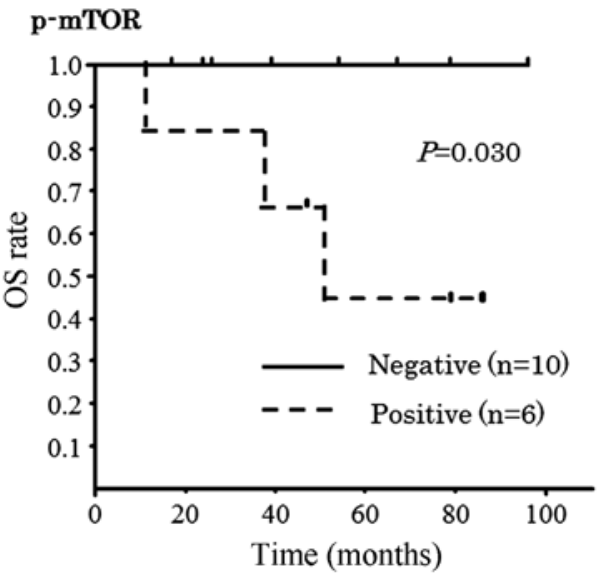

Figure 5. Overall survival (OS) in accordance with the presence of p-mTOR. Significant difference $(\mathrm{P}=0.029)$ was noted.

compared with that of pancreatic cancer; this was reported in our previous study (20). Given the lower expression pattern of HLA class I in PNETs compared with pancreatic carcinoma, it may be speculated that the extent of TILs in PNETs is not sufficiently adequate to serve as an independent prognosticator.

In terms of molecular biomarkers such as ATRX, DAXX, mTOR and p-mTOR, there were statistically significant correlations between DAXX, p-mTOR and PHR in the present study. In addition, lower ATRX expression, lower DAXX expression and higher $\mathrm{p}$-mTOR expression were found to be significant prognostic factors for DFS. de Wilde et al suggested that loss of nuclear expression of ATRX and/or DAXX, occurring in MEN1 neuroendocrine tumors, is correlated with the existence of the alternative lengthening of telomeres (ALT) phenotype. The present study also showed that loss of nuclear expression of ATRX and/or DAXX and the presence of the ALT phenotype occurred only in larger $(\geq 3 \mathrm{~cm})$ PNETs, and these changes are late events in PNETs (14). Marinoni et al showed that loss of DAXX or ARTX protein and ALT are associated with chromosomal instability in PNETs. In addition, loss of DAXX or ARTX was found to be correlated with tumor stage and metastasis, DFS and OS (21). In terms of p-mTOR, Han et al suggested that high expression of p-mTOR and low expression of the PTEN group portended worse prognosis than low expression of p-mTOR and high expression of PTEN, but did not estimate worse prognosis in high expression of p-mTOR only (18). In the present study, we found that the expression patterns of ATRX, DAXX and p-mTOR were correlated with DFS, while only p-mTOR was found to correlate with OS.

In other malignancies, earlier introduction of postoperative adjuvant therapy has been proven to improve patient prognosis based on such clinical risk factors as node metastasis, human epidermal growth factor receptor 2 (HER2) status, and epidermal growth factor receptor (EGFR) expression level $(22,23)$. Collectively, this evidence, along with the data from the present study, supports a strategy to introduce cytostatic drugs such as mTOR, sunitinib or ocreotide immediately following curative resection of the primary tumor site, based on the risk factors for disease recurrence identified by the expression patterns of the various molecular markers investigated in the present study.
The present investigation was limited by its retrospective study design and the small number of study patients. Further exploratory studies concerning the prediction of the recurrence of PNETs are warranted.

In conclusion, ATRX, DAXX and p-mTOR are useful molecular biomarkers for predicting PHR in patients who undergo radical surgery for PNETs. Use of these biomarkers will enable earlier decisions on which patients may benefit from adjuvant therapy.

\section{Acknowledgements}

The authors thank Hiraku Shida for his technical assistance in the immunohistochemical analysis.

\section{References}

1. Metz DC and Jensen RT: Gastrointestinal neuroendocrine tumors: pancreatic endocrine tumors. Gastroenterology 135: 1469-1492, 2008.

2. de Wilde RF, Edil BH, Hruban RH and Maitra A: Welldifferentiated pancreatic neuroendocrine tumors: from genetics to therapy. Nat Rev Gastroenterol Hepatol 9: 199-208, 2012.

3. Panzuto F, Nasoni S, Falconi M, et al: Prognostic factors and survival in endocrine tumor patients: comparison between gastrointestinal and pancreatic localization. Endocr Relat Cancer 12: 1083-1092, 2005.

4. Yao JC, Hassan M, Phan A, et al: One hundred years after 'carcinoid': epidemiology of and prognostic factors for neuroendocrine tumors in 35,825 cases in the United States. J Clin Oncol 26: 3063-3072, 2008.

5. Bloomston M, Muscarella $\mathrm{P}$, Shah MH, et al: Cytoreduction results in high perioperative mortality and decreased survival in patients undergoing pancreatectomy for neuroendocrine tumors of the pancreas. J Gastrointest Surg 10: 1361-1370, 2006.

6. Kobayashi N, Hiraoka N, Yamagami W, et al: FOXP3 ${ }^{+}$regulatory $T$ cells affect the development and progression of hepatocarcinogenesis. Clin Cancer Res 13: 902-911, 2007.

7. Galon J, Costes A, Sanchez-Cabo F, et al: Type, density, and location of immune cells within human colorectal tumors predict clinical outcome. Science 313: 1960-1964, 2006.

8. Jiao Y, Shi C, Edil BH, et al: DAXX/ATRX, MEN1, and mTOR pathway genes are frequently altered in pancreatic neuroendocrine tumors. Science 331: 1199-1203, 2011.

9. Nave BT, Ouwens M, Withers DJ, Alessi DR and Shepherd PR: Mammalian target of rapamycin is a direct target for protein kinase $\mathrm{B}$ : identification of a convergence point for opposing effects of insulin and amino-acid deficiency on protein translation. Biochem J 344: 427-431, 1999.

10. Wullschleger S, Loewith R and Hall MN: TOR signaling in growth and metabolism. Cell 124: 471-484, 2006.

11. Yao JC, Shah MH, Ito T, et al: Everolimus for advanced pancreatic neuroendocrine tumors. N Engl J Med 364: 514-523, 2011.

12. Crippa S, Partelli S, Boninsegna L and Falconi M: Implications of the new histological classification (WHO 2010) for pancreatic neuroendocrine neoplasms. Ann Oncol 23: 1928, 2012.

13. Cho Y, Miyamoto M, Kato K, et al: $\mathrm{CD}^{+}$and $\mathrm{CD}^{+} \mathrm{T}$ cells cooperate to improve prognosis of patients with esophageal squamous cell carcinoma. Cancer Res 63: 1555-1559, 2003.

14. de Wilde RF, Heaphy CM, Maitra A, et al: Loss of ATRX or DAXX expression and concomitant acquisition of the alternative lengthening of telomeres phenotype are late events in a small subset of MEN-1 syndrome pancreatic neuroendocrine tumors. Mod Pathol 25: 1033-1039, 2012.

15. La Rosa S, Klersy C, Uccella S, et al: Improved histologic and clinicopathologic criteria for prognostic evaluation of pancreatic endocrine tumors. Hum Pathol 40: 30-40, 2009.

16. Scarpa A, Mantovani W, Capelli P, et al: Pancreatic endocrine tumors: improved TNM staging and histopathological grading permit a clinically efficient prognostic stratification of patients. Mod Pathol 23: 824-833, 2010.

17. Katz SC, Donkor C, Glasgow K, et al: $\mathrm{T}$ cell infiltrate and outcome following resection of intermediate-grade primary neuroendocrine tumours and liver metastases. HPB 12: 674-683, 2010. 
18. Han X, Ji Y, Zhao J, Xu X and Lou W: Expression of PTEN and mTOR in pancreatic neuroendocrine tumors. Tumour Biol 34: 2871-2879, 2013.

19. Ino Y, Yamazaki-Itoh R, Shimada K, et al: Immune cell infiltration as an indicator of the immune microenvironment of pancreatic cancer. Br J Cancer 108: 914-923, 2013.

20. Tsuchikawa T, Hirano S, Tanaka E, et al: Novel aspects of preoperative chemoradiation therapy improving anti-tumor immunity in pancreatic cancer. Cancer Sci 104: 531-535, 2013.

21. Marinoni I, Kurrer AS, Vassella E, et al: Loss of DAXX and ATRX are associated with chromosome instability and reduced survival of patients with pancreatic neuroendocrine tumors. Gastroenterology 146: 453-460.e5, 2014.
22. Inada S, Koto T, Futami K, Arima S and Iwashita A: Evaluation of malignancy and the prognosis of esophageal cancer based on an immunohistochemical study (p53, E-cadherin, epidermal growth factor receptor). Surg Today 29: 493-503, 1999.

23. Garcia I, Vizoso F, Martin A, et al: Clinical significance of the epidermal growth factor receptor and HER2 receptor in resectable gastric cancer. Ann Surg Oncol 10: 234-241, 2003. 